[6] L. C. Janse. Chem. Weekbl. 1946, 42 (41), 284.

[7] Tamsma, Janssen, Passenier. Netcherlands Milk and Dairy Journal. Avril 1947, 78.

[8] G. Roeder. Zeitsch. f. Lebensm. Untersuch. und Forsch., 1948, 88, 461.

[9] L. Radema et H. Mulder. Netherlands Milk and Dairy Journal. 1951, 104.

[10] P. Macherel. Le Lait. 1948, 515.

[11] Netherlands Milk and Dairy Journal. 1952, (6), 62.

[12] Milchwissenschaft. Juin 1953, 237. (D.I.N., 12.837.)

[I3] Ch. Brioux. Le Lait. 1926, 6, 766.

[14] G. Chaineux et P. Simonart. Agricultura. Louvain. Septembre 1938.

[15] H. Hostettler et H. Hann. Mitteil. Lebensm. und Hyg., 1947, (38), 354.

[16] CAdor et Macherel, Le Lait. 1945, 289.

[17] G. Roeder. Milchwirtsch. Forschung. 1928, 258.

[18] G. Roeder. Zeitsch. f. Lebensm. Untersuch. und Forsch. 1948, 88, 461.

[19] G. Roeder. Milchwirtschaft. Forch. 1940, 20, 200.

[20] J. PIEN. Conditions à remplir par les pipettes à lait pour butyrométrie. C.R. du Congrès. Section III. Question 4.

[21] J. PIEN. Appareil à projection pour la lecture précise des butyromètres. C.R. du Congrès. Section III. Question 4.

\title{
LES LIMITES DE VARIATION DES INDICES DE LA MATIËRE GRASSE DU BEURRE (1)
}

par

\section{SImonne KUZDZAL-SAVOIE}

Chargée de Recherches à l'Institut National de la Recherche Agronomique

Deux études successives poursuivies ces dernières années, l'une sur les variations saisonnières de l'indice d'iode du beurre [1], l'autre sur l'influence du stade de lactation [2], nous ont permis de trouver que les limites extrêmes, généralement admises en France, des indices du beurre pur, sont trop restreintes.

Nous envisagerons successivement les indices d'iode, de saponification, l'acidité volatile soluble et insoluble.

\section{Indice d'iode}

En France, les valeurs extrêmes de 26 et 36 rapportées par Rochatx et TAPERnoux [3] servent généralement de base lors de l'interprétation des résultats d'analyses.

D'après HUNZiker [4], la moyenne générale est de 32 environ. Les variations usuelles s'échelonnent de 30 à 34 et les variations extrêmes de 26 à 38 .

(1) An. fals. et fr., 1956, n $\mathrm{n}^{\circ} 575,452$. 
Cox et McDowaLl [5] travaillant sur des échantillons mensuels de beurre fabriqués dans neuf laiteries de Nouvelle-Zélande, constatent qu'au cours de quatre années consécutives l'indice d'iode moyen varie de 34,0 à 40,2 .

D'après Bergman et Joost [6], la moyenne des indices d'iode du beurre de différentes laiteries de Suède varie de 30,4 (décembre) à 39,0 (septembre) avec un indice d'iode minimum de 26,5 et maximum de 43,0 .

Ces mêmes auteurs ont suivi dans une laiterie du Nord de la Suède les variations de l'indice d'iode de la matière grasse du lait fourni par chaque producteur. Les valeurs extrêmes trouvées sont de 25,1 et 47,2 .

Sohwartz et Mumm [7], travaillant sur la matière grasse du lait de mélange de vingt troupeaux de vaches du Schleswig-Holstein (race Pie noire de Plaine) indiquent les chiffres suivants : indice d'iode minimum 27,5 (février) et maximum 48,1 (septembre).

Sur un total de 473 déterminations effectives sur la matière grasse de laits de différentes vaches, ECKLES et SHAW [8] constatent que les indices d'iode extrêmes sont 23,8 et 49,4 . La répartition en pourcentage est la suivante :

\begin{tabular}{|c|c|c|}
\hline De 20 à 25 & $\ldots$ & 0,4 \\
\hline De 25 à 30 & $\ldots \ldots \ldots \ldots \ldots \ldots$ & 21,5 \\
\hline De 30 à 35 & $\ldots \ldots \ldots \ldots \ldots$ & 47,4 \\
\hline De 35 à 40 & $\ldots \ldots \ldots \ldots \ldots \ldots$ & 24,9 \\
\hline De 40 à 45 & 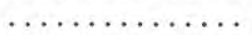 & 5,4 \\
\hline De 45 à 50 & $\ldots \ldots \ldots \ldots \ldots$ & 0,4 \\
\hline
\end{tabular}

Utilisant la méthode de WiJs telle qu'elle est décrite par GRossFELD [9] nous avons étudié au cours des années 1950-1951-1952, les variations de l'indice d'iode de deux beurres : l'un fabriqué en Normandie (Bayeux), l'autre fabriqué en Alsace (Riedseltz).

Les valeurs extrêmes trouvées sont : 28,6 (janvier 1950 à Riedseltz) et 44,2 (avril 1952 à Bayeux).

L'étude poursuivie en 1953-1954 sur le beurre de Poligny (Jura) conduit aux indices extrêmes de 28,3 (février 1953) et 46,7 (octobre 1953) et sur la matière grasse du lait de mélange du troupeau de Jouy-en-Josas aux indices extrêmes suivants :

$\begin{array}{rlr}\text { Minima }: 28,0 & 24 \text { février } & 1953 \\ 26,2 & 25 \text { janvier } & 1954 \\ \text { Maxima }: 44,4 & 13 \text { septembre } & 1954 \\ 44,8 & 23 \text { septembre } & 1953\end{array}$

Des indices d'iode particulièrement élevés ont été trouvés au 
cours de l'analyse de la matière grasse de laits individuels provenant de vaches de différentes étables.

Le tableau suivant indique ces résultats :

\begin{tabular}{|c|c|c|c|c|c|}
\hline Lieu & $\begin{array}{l}\text { Nom } \\
\text { de la } \\
\text { vache }\end{array}$ & Race & $\begin{array}{c}\text { Date } \\
\text { de } \\
\text { vêlage }\end{array}$ & $\begin{array}{l}\text { Date de } \\
\text { prélè. } \\
\text { vement }\end{array}$ & $\begin{array}{l}\text { Indice } \\
\text { d'iode }\end{array}$ \\
\hline $\begin{array}{c}\text { Trappes } \\
\text { ferme } \\
\text { E. Cuypers }\end{array}$ & Première & Hollandaise & $3-3-52$ & $\begin{array}{l}21-3-52 \\
21-4-52 \\
20-6-52 \\
23-5-52\end{array}$ & $\begin{array}{l}48,7 \\
57,1(1) \\
55,2 \\
51,4\end{array}$ \\
\hline $\begin{array}{l}\text { Vaux- } \\
\text { sur-Poligny } \\
\text { ferme } \\
\text { Laubier }\end{array}$ & $\begin{array}{l}\text { Rêveuse } \\
\text { Sirène }\end{array}$ & $\begin{array}{l}\text { Pie rouge } \\
\text { Pie rouge }\end{array}$ & $\begin{array}{l}16-2-53 \\
16-2-53\end{array}$ & $\begin{array}{l}28-10-53 \\
3-10-53\end{array}$ & $\begin{array}{l}58,0 \\
58,6\end{array}$ \\
\hline $\begin{array}{c}\text { Jouy } \\
\text { (C.N.R.Z.) }\end{array}$ & $\begin{array}{l}\text { Eglantine } \\
\text { Astrée } \\
\text { Baby } 101\end{array}$ & $\begin{array}{l}\text { Hollandaise } \\
\text { Normande } \\
\text { Normande }\end{array}$ & $\begin{array}{c}12-2-53 \\
6-4-53 \\
27-10-53\end{array}$ & $\begin{array}{l}21-6-53 \\
27-9-53 \\
27-9-53 \\
28-10-54\end{array}$ & $\begin{array}{l}50,3 \\
49,4 \\
47,7 \\
50,3\end{array}$ \\
\hline
\end{tabular}

Les indices d'iode déterminés au cours de l'étude sur l'influence du stade de lactation sur la composition de la matière grasse [2] peuvent être répartis de la façon suivante :

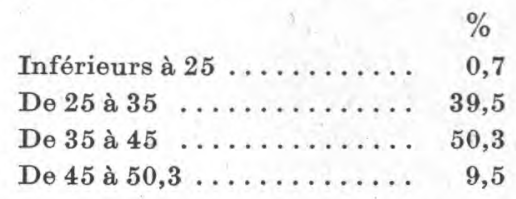

Ainsi près de $60 \%$ des indices d'iode trouvés au cours de cette expérience dépassent la valeur maxima généralement admise en en France.

\section{Indice de saponification}

L'indice de saponification ou nombre de Köttstorffer est le nombre de milligrammes d'hydrate de potassium nécessaires pour effectuer la saponification complète de 1 gramme de corps gras.

(1) Nourriture à l'herbe exclusivement. 
RochaIx et TAPERNOUX [3] signalent un indice de saponification moyen de 225 et admettent les valeurs extrêmes de 220-222 et 232-235, voisines de celles rapportées par HuNzIKER [4].

Au cours de l'étude poursuivie pendant quatre années sur neuf beurres de Nouvelle-Zélande, Cox et McDowalL [5] trouvent un indice de saponification minimum de 219,8 et maximum de 234,4 .

EckLEs et SHaw [8], aux Etats-Unis, sur des laits individuels de vaches de différentes races trouvent à partir de 488 résultats un indice minimum de 200,1 et maximum de 252,3 , avec cependant, $81 \%$ des indices entre 220 et 235 .

Utilisant la méthode officielle de détermination de l'indice de saponification telle qu'elle est décrite par BRUNo [10 a] nous avons suivi les variations saisonnières du beurre de Poligny (Jura) et du beurre fabriqué à partir du lait de mélange de Jouy-en-Josas, au cours des années 1953-1954 [2]. L'amplitude des variations, plus accentuée à Jouy-en-Josas, qu'à Poligny, s'étend de 222, 1 (10 novembre 1953) à 244,2 (31 mars 1953).

Sur un total de 145 déterminations effectuées sur la matière grasse de laits prélevés au cours des lactations de 4 vaches du troupeau de Jouy-en-Josas, on obtient la répartition suivante :

\begin{tabular}{|c|c|}
\hline Inférieurs à $220 \ldots \ldots \ldots \ldots$ & 4,1 \\
\hline De 220 à $230 \ldots$ & 54,5 \\
\hline De 230 à $240 \ldots \ldots \ldots \ldots$ & 35,9 \\
\hline Supérieurs à $240 \ldots \ldots \ldots$ & 5,5 \\
\hline
\end{tabular}

avec un minimum de 216,7 (Alcippe, le 15 avril 1954) et un maximum de 245,5 (Eglantine, le 6 avril 1953).

Ainsi, les variations de l'indice de saponification peuvent être plus amples que celles habituellement retenues en France.

\section{Acidité volatile}

Nous envisagerons successivement l'acidité volatile soluble et l'acidité volatile insoluble.

Acidité volatile soluble : ou nombre de millilitres de solution alcaline décinormale nécessaires pour neutraliser les acides volatils solubles de 5 grammes de matière grasse.

Pour Rochaix et Tapernoux [3], l'acidité volatile soluble (indice R.M. W.) est toujours au minimum égale à 28. HALPHEN [11] indique cependant des variations moyennes de 22,7 à 31,0 et note que le procédé officiel de détermination des acides volatils, procédé LEAFMANN-BEAM [10b], donne des résultats légèrement inférieurs $\left(1 / 10^{\mathrm{e}}\right)$ aux indices de REICHERT. 
Hunziker [4] signale des indices de Reichert du beurre variant de 24 à 34 .

Richmond [12a] sur 700 échantillons trouve des indices de Reichert variant de 21,2 à 35,0 .

Cox et McDow ALL [5்] signalent des valeurs extrêmes de 23,1 et 33,9 en Nouvelle-Zélande.

L'indice de Reichert d'après ScHWARz et MUMm [7] serait minimum en août-septembre $(25,8)$ et maximum en mars $(32,2)$. Un rapport $d u$ laboratoire du Comité de Lancashire signalé par RICHMOND [12b] aboutit à des conclusions très voisines.

EckLes et SHAw [8] obtiennent la répartition suivante à partir de 484 déterminations d'indice de Reichert :

\begin{tabular}{|c|c|}
\hline 5 à 10 & $\ldots \ldots \ldots \ldots \ldots \ldots$ \\
\hline 10 à 15 & $\ldots \ldots \ldots \ldots \ldots \ldots$ \\
\hline 15 à 20 & $\ldots \ldots \ldots \ldots \ldots \ldots$ \\
\hline 20 à 25 & $\ldots \ldots \ldots \ldots \ldots \ldots \ldots$ \\
\hline 25 à 30 & $\ldots \ldots \mu_{1}$ \\
\hline 30 à 35 & $\ldots \ldots \ldots, \ldots, \cdots$ \\
\hline
\end{tabular}

Ces auteurs [8] rappellent que Richmond considère que les beurres ayant un indice de Reichert inférieur à 25 doivent être considérés comme suspects et que la limite inférieure absolue doit être fixée à 20. Or les résultats de EckLEs et SHAw [8] montrent que $41,1 \%$ des déterminations sont inférieures ou égales à 25 et $7,5 \%$ sous la limite absolue fixée par Richmond.

Les variations de l'acidité volatile soluble que nous avons suivies, par la méthode officielle française, tant à Jouy qu'à Poligny, confirment le mouvement saisonnier indiqué par ScHWARz et Mumm [7].

Les déterminations de l'acidité volatile soluble de la matière grasse des laits des quatre vaches étudiées à Jouy pendant une lactation entière et le premier mois de la lactation suivante, peuvent être groupées de la façon suivante :

Sur un total de 136 déterminations :

\begin{tabular}{|c|c|}
\hline Inférieures à $20 \ldots \ldots \ldots \ldots$ & 0,7 \\
\hline De 20 à $25 \ldots \ldots \ldots \ldots \ldots$ & 14,0 \\
\hline De 25 à $30 \quad \ldots \ldots \ldots \ldots \ldots$ & 41,3 \\
\hline De 30 à $35 \quad \ldots \ldots \ldots \ldots \ldots$ & 32,3 \\
\hline De 35 à $40 \quad \ldots \ldots \ldots \ldots \ldots$ & 11,0 \\
\hline Supérieures à $40 \ldots \ldots \ldots \ldots$ & 0,7 \\
\hline
\end{tabular}

Ainsi près de $40 \%$ de nos résultats se situent au-dessus du chiffre maximum retenu par HALPHEN [11] et environ $20 \%$ restent inférieurs à 28 . 
Acidité volatile insoluble: c'est le nombre de millilitres de solution alcaline décinormale nécessaires pour neutraliser les acides gras volatils insolubles de 5 grammes de matière grasse. L'indice de Polenske [12c] diffère très légèrement de la détermination de l'acidité volatile insoluble par la méthode officielle franȩaise [10c].

RochaIx et TAPeRnoux [3] souvent consultés signalent une variation de 2 à 2,5 au-dessus de laquelle la fraude peut être suspectée.

BRUNo [10d] rapporte cependant des résultats supérieurs communiqués par la Station Agronomique d'Arras (2,3 à 4,2).

Les variations saisonnières de l'indice de Polenske étudiées par Schwarz et Mumm [7] s'étendent de 1,5 à 2,8 .

Par la méthode officielle française, nous avons suivi les variations saisonnières de l'acidité volatile insoluble des beurres de Poligny (Jura) et de la matière grasse du lait de mélange de Jouyen-Josas. Le mouvement saisonnier est comparable à celui indiqué par Schw ARz et MUмm [7] avec un minimum en septembre et octobre et maximum fin hiver-printemps, mais les valeurs que nous trouvons sont plus élevées :

2,0 à 4,9 (Poligny)

2,2 à 5,8 (Jouy).

A partir des résultats trouvés à Jouy-en-Josas, au cours de l'étude sur l'influence du stade de lactation, nous obtenons (sur 136 déterminations) :

\begin{tabular}{lllr} 
& & & \multicolumn{1}{c}{$\%$} \\
Inférieurs à $1,5 \ldots \ldots \ldots \ldots$ & \multicolumn{1}{c}{0,7} \\
De 1,5 à 2,5 & $\ldots \ldots \ldots \ldots \ldots$ & 15,4 \\
De 2,5 à 3,5 & $\ldots \ldots \ldots \ldots \ldots$ & 41,9 \\
De 3,5 à 4,5 & $\ldots \ldots \ldots \ldots$ & 28,8 \\
De 4,5 à 5,5 & $\ldots \ldots \ldots \ldots \ldots$ & 12,5 \\
Supérieurs à 5,5 & $\ldots \ldots \ldots \ldots$ & 0,7
\end{tabular}

D'une façon générale à une acidité volatile soluble élevée correspond une acidité volatile insoluble élevée. Le rapport $\frac{\text { AVI }+100}{\text { AVS }}$ n'est toutefois pas constant. Richmond [12d] signale pour un même indice de Reichert une marge de variations de l'indice de Polenske qui nous semble un peu étroite.

D'après les résultats que nous avons obtenus sur le beurre de Poligny ou de Jouy-en-Josas ou sur les laits individuels nous constatons une grande variabilité du rapport $\frac{A V I \times 100}{A V S}$. CHOLLET 
et Camus [13] signalent que ce rapport varie de 8 à 15. Les mêmes limites de ce rapport sont indiquées par RochaIX et TAPERNoux [3], qui, cependant, retiennent pour l'acidité volatile soluble, la valeur moyenne de 28 et pour l'acidité volatile insoluble une valeur variant de 2 à 2,5 . C'est à peu de choses près dans ces limites de 8 à 15 que varie le rapport $\frac{\text { AVI } \times 100}{\text { AVS }}$ calculé à partir de toutes les valeurs d'acidité volatile que nous avons déterminées.

\section{Discussion}

Il apparaît hors de doute que les limites extrêmes des indices généralement admises en France sont trop étroites, sauf cependant, celles indiquées pour le rapport $\frac{\operatorname{AVI} \times 100}{\mathrm{AVS}}$.

Il est évident que les plus grandes variations sont observées sur la matière grasse de laits individuels. Cependant, même pour les beurres fabriqués à partir d'une crème de grand mélange, nous avons observé une grande variabilité des indices, au cours de l'année et d'une région de France à une autre. Lorsqu'un tel beurre subit l'analyse en vue de déterminer s'il est de qualité marchande, il est recommandable d'analyser simultanément un beurre de la région fabriqué à la même période et présentant toutes garanties de pureté. Comme le souligne BRUNo [10e] "pour formuler un avis sur la pureté d'un beurre il ne suffit pas de bien savoir ce qu'il est, il importe tout autant de savoir ce qu'il doit être ".

La composition de la matière grasse du lait varie non seulement en fonction de la saison mais également en fonction du stade de lactation et d'autres facteurs tels que la race, la nature du sol, etc... Nous avons vu [2] qu'en fin de lactation l'indice d'iode s'élève alors que s'abaissent nettement les indices de saponification et d'acidité volatile. Or, sur la détermination de ces derniers indices repose en partie la conclusion de l'expert. Dans les grandes régions beurrières de France (Normandie, Charente, Est), l'étalement des vêlages est de plus en plus recherché ; toute l'année les laits de fin de lactation ne constituent ainsi qu'une mineure partie de la totalité du lait ramassé par une laiterie de grande ou de moyenne importance. Leur présence n'entraîne généralement pas de perturbations dans la nature du beurre fabriqué. Il n'en est pas de même pour un beurre fabriqué dans une laiterie dont la zone de ramassage est restreinte ou plus encore pour un beurre fermier pour lequel il est recommandable avant d'établir le rapport d'analyse, de s'informer du stade de lactation moyen des vaches du troupeau et de l'étable considéré. 


\title{
BIBLIOGRAPHIE
}

[1] M. Bejambes et S. Savoie. Chim. et Ind., 71, 3, 501-506.

[2] S. Kuzdzal-Savole. Ann. Tech. I.N.R.A., 1956, série E, no 1, 1.

[3] A. Rochaix et A. Tapernoux. 1948. Vigot Frères. Paris.

[4] O. Hunziker. La Grange, Illinois. 1927, p. 584.

[5] C. A. Cox et P. H. McDowALL. Journal Dairy Res., 1948, 25, 377.

[6] T. Bergman et Kr. Joost. XIIIth Intern. Dairy Congr. The Hague. June 22-26. Vol. II, sect. I, Subj. 1, p. 89, 1953.

[7] G. Schwarz et H. Mumm. Kieler Milchwirtschaftl. Forschungsberichte V Band. 1953.

[8] C. H. Eokles et R. H. Shaw. Bureau of Anim. husb. 1913, bull. 156.

[9] J. Grossfeld. Handbuch der Lebensmittel Chem. IV Band, Fette und Oele. Verlag von Julius Springer. Berlin 1939.

[10] A. BRuno. 10a, p. 48 ; 10b, p. 44;10e, p. 46 ; 10d, p. $152 ; 10 \mathrm{e}$, p. 166.

[11] G. Halphen. 1912. Ch. Béranger. Paris, p. 137.

[12] H. D. Richmond, G. D. Elsdon et G. H. Walker. Dairy Chem., Ch. Griffin and Co Ltd, 1942, 12a p. 176;12b, p. 178; 12c, p. 403 ; 12 d, p. 179.

[13] A. Chollet et A. Camus. Le Lait. 1937, 135.

\section{REVUE}

\section{L'AUTOMATION DANS L'INDUSTRIE LAITIËRE}

\author{
par \\ G. GÉNIN
}

Ingénieur E.P.C.

Automatisme, automaticité, l'automatique, l'automation, l'automatisation, sont des termes que l'on trouve aujourd'hui fréquemment cités dans la littérature technique et qui, en attendant qu'une terminologie française se soit établie, sont utilisés pour définir un nouveau progrès technologique conduisant à la mécanisation plus poussée des usines.

Si l'on ouvre un dictionnaire dans l'espoir d'y trouver la définition de ces termes, on ne trouve rien qui corresponde exactement à leur signification actuelle et d'ailleurs, la signification qu'on en donne est souvent différente suivant qu'on s'adresse à un technicien ou un autre. Cependant, sans vouloir faire œuvre de linguiste, on peut dire que l'automatisme est le perfectionnement que vient de réaliser l'industrie et qui permet de passer de ce qu'on a appelé la mécanisation proprement dite à la commande des installations mécanisées. On peut dire encore que la mécanisation était simple- 